less than those given in Table I. Table II gives the value of $s$ corresponding to each value of $k$.

However this method of producing solutions to (A) with a small number of terms is subject to the following weakness. We had assumed that from any particular solution to (A) solutions of higher degree would be generated containing the least number of terms $s$, so long as the most frequent difference $d$ was used at each step. After producing the following results this assumption was seen to be false.

When forming Table I the multiplier $(1-x)$ was used with $\prod_{j=1}^{11}\left(1-x^{j}\right)$ to produce a solution to the extended problem where $s=22$ for $k=11$. This is equivalent to starting with the solution $0,2 \stackrel{1}{=} 1,1$ and using Theorem 2 with $d=2,3, \cdots, 11$. Table III compares the lengths of the solutions generated in this manner with those generated from the same initial solution but using the most frequent difference $d$ at each step.

Thus, by a more careful choice of $d$, the length of solutions can be decreased for $k=6,7,8,9,10$. But for $k=11$ this produces a solution to the extended problem where $s=24$. This solution is longer than that obtained from a sequence of solutions which was constructed from values for $d$ that did not always represent the most frequent difference.

Finally, although solutions to (A) for $k=6$ and $s=7$ exist, we proved that no such solution can be obtained from a sequence generated by any solution for $k=1$ and $s=2$ using the most frequent difference $d$ at each step.

Although Theorem 2 was used to generate most solutions for $k \leqq 9$ where $s=k+1$, it appears that for $k \geqq 10$ it alone will not be sufficient.

3. Acknowledgments. The author is indebted to Dr. Z. A. Melzak for his suggestions and helpful criticisms.

University of Victoria

Victoria, British Columbia

1. L. Bastien, Sphinx-Oedipe, v. 8, 1913, pp. 171-172.

2. M. Frolov, Sphinx-Ocdipe, v. 4, 1909, pp. 81-89.

3. G. H. HARDY \& E. M. Wright, An Introduction to the Theory of Numbers, 4 th ed., Clarendon Press, Oxford, 1960.

4. Z. A. Melzak, "A note on the Tarry-Escott problem," Canad. Math. Bull., v. 4, 1961, pp. 233-237.

5. G. TARRY, L'Intermédiaire des Mathematiciens, v. 19, 1912, pp. 219-221.

6. E. M. WrIGHT, "On Tarry's problem. I," Quart. J. Math., v. 6, 1935, pp. 261-267.

\title{
Numerical Solutions of the Diophantine Equation
}

$$
y^{3}-x^{2}=k
$$

\section{By M. Lal, M. F. Jones and W. J. Blundon}

Introduction. The distribution of squares and cubes differing by a given integer is very interesting [1] and has attracted many mathematicians over the past few centuries. Probably this is due to the fact that $y^{3}-x^{2}=k$ is the simplest of all nontrivial Diophantine equations of degree greater than two. The solution of this equation is equivalent to the problem of representation of numbers by binary cubic

Received September 7, 1965. 
TABLE 1

Values of $|k|$ with No. of Solutions $\geqq 6$

\begin{tabular}{|c|c|c|c|c|c|c|c|c|}
\hline$K$ & $N+$ & $N-$ & $K$ & $N+$ & $N-$ & $K$ & $N+$ & $N-$ \\
\hline 17 & 0 & 8 & 1737 & 0 & 11 & 4977 & 0 & 8 \\
\hline 73 & 0 & 6 & 1792 & 3 & 3 & 5328 & 0 & 6 \\
\hline 100 & 3 & 6 & 1809 & 0 & 6 & 5400 & 0 & 8 \\
\hline 113 & 0 & 6 & 1872 & 0 & 7 & 5543 & 6 & 0 \\
\hline 207 & 7 & 0 & 1900 & 0 & 6 & 5696 & 3 & 5 \\
\hline 225 & 0 & 13 & 1999 & 6 & 0 & 5841 & 0 & 10 \\
\hline 252 & 1 & 5 & 2052 & 0 & 6 & 5887 & 4 & 2 \\
\hline 297 & 0 & 9 & 2071 & 6 & 0 & 6236 & 6 & 1 \\
\hline 316 & 1 & 7 & 2089 & 0 & 14 & 6400 & 4 & 7 \\
\hline 343 & 5 & 2 & 2188 & 5 & 1 & 6479 & 6 & 0 \\
\hline 368 & 7 & 1 & 2241 & 0 & 6 & 6625 & 0 & 6 \\
\hline 388 & 0 & 6 & $2 ; 12$ & 1 & 5 & 6908 & 5 & 1 \\
\hline 431 & 9 & 0 & 2351 & 10 & 0 & 6921 & 0 & 6 \\
\hline 496 & 6 & 1 & 2600 & 1 & 5 & 7057 & 0 & 11 \\
\hline 503 & 8 & 0 & 2628 & 0 & 9 & 7100 & 5 & 4 \\
\hline 512 & 1 & 5 & 2817 & 0 & 10 & 7232 & 0 & 8 \\
\hline 516 & 4 & 3 & 3025 & 0 & 11 & 7353 & 0 & 7 \\
\hline 568 & 0 & 7 & 3033 & 0 & 8 & 7568 & 0 & 8 \\
\hline 648 & 6 & 0 & 3332 & 6 & 2 & 7600 & 4 & 2 \\
\hline 676 & 6 & 1 & 3356 & 0 & 6 & 7785 & 0 & 8 \\
\hline 775 & 7 & 2 & 3592 & 1 & 5 & 7804 & 7 & 0 \\
\hline 828 & 5 & 1 & 3664 & 0 & 8 & 7948 & 0 & 6 \\
\hline 847 & 7 & 0 & 3807 & 11 & 0 & 8036 & 3 & 4 \\
\hline 873 & 0 & 9 & 3844 & 0 & 8 & 8225 & 0 & 11 \\
\hline 892 & 1 & 5 & 3896 & 11. & 0 & 8281 & 0 & 9 \\
\hline 964 & 4 & 3 & 3969 & 0 & 9 & 8289 & 0 & 7 \\
\hline 999 & 6 & 0 & 4032 & 2 & 4 & 8433 & 0 & 6 \\
\hline 1016 & 0 & 7 & 4087 & 7 & 0 & 8532 & 7 & 0 \\
\hline 1025 & 0 & 16 & 4112 & 0 & 10 & 8623 & 5 & 2 \\
\hline 1071 & 6 & 0 & 4220 & 6 & 0 & 8673 & 0 & 6 \\
\hline 1088 & 0 & 9 & 4312 & 0 & 10 & 8676 & 1 & 9 \\
\hline 1225 & 1 & 5 & 4329 & 0 & 7 & 8712 & 6 & 0 \\
\hline 1304 & 0 & 6 & 4356 & 0 & 8 & 8900 & 1 & 12 \\
\hline 1305 & 0 & 9 & 4481 & 0 & 12 & 9052 & 1 & 5 \\
\hline 1439 & 8 & 0 & 4598 & 4 & 2 & 9297 & 0 & 8 \\
\hline 1712 & 6 & 1 & 4600 & 1 & 5 & 9559 & 7 & 1 \\
\hline 1724 & 5 & 1 & 4672 & 0 & 7 & 9748 & 9 & 0 \\
\hline 1727 & 7 & 0 & 4799 & 7 & 0 & 9936 & 0 & 6 \\
\hline 1729 & 1 & 5 & 4825 & 0 & 7 & 9967 & 6 & 0 \\
\hline
\end{tabular}

forms [2]. Thus the solution of the indeterminate equation of third degree

$$
y^{3}-x^{2}=k
$$

is equivalent to solving a finite number of equations $(a, b, c, d)=1$, where $(a, b, c, d)$ is a binary cubic form.

At present, very little is known about the theory of binary cubic forms. In this respect, the theory for negative discriminants is better developed and for $0<-k \leqq 100$, all solutions of (1) have been found [3]. However for positive discriminants, progress has been rather slow and for the equivalent positive range, 
TABLE 2

\begin{tabular}{rr|r|r}
\hline & & \multicolumn{2}{|c}{ Number of solutions } \\
\cline { 3 - 4 } & & Positive $K$ & Negative $K$ \\
\hline & & 0 & 1774 \\
& $0 \leqq y<0$ & 2140 & 2669 \\
& $10^{2} \leqq y<10^{3}$ & 712 & 649 \\
& $10^{3} \leqq y<10^{4}$ & 294 & 185 \\
& $10^{4} \leqq y<10^{5}$ & 64 & 66 \\
& $10^{5} \leqq y<10^{6}$ & 13 & 23 \\
& $10^{6} \leqq y<4.64 \times 10^{6}$ & 0 & 4 \\
\hline Total & & 3223 & 5370 \\
\hline
\end{tabular}

20 cases remain to be resolved [3], [4]. For $|k|>100$, it appears that complete solutions are lacking. It was therefore felt desirable to obtain solutions of (1) by means of a numerical search.

This search was conducted with

$$
\begin{gathered}
|k| \leqq 9999, k \neq 0 \text { and } \\
0 \leqq x<10^{10} .
\end{gathered}
$$

These parameters fix the range of $y$ to be

$$
-21 \leqq y<y_{\max } ; \quad y_{\max }=4,641,589 \text {. }
$$

We anticipate that the results of such an extensive search will be useful for checking some of the conjectures concerning this equation and also provide further insight into the theory of binary cubic forms.

Method. By rewriting (1) as

$$
x^{2}=y^{3}-k,
$$

then, for a given $y, x$ is bounded by

$$
x_{\min }<x<\left(y^{3}+9999\right)^{1 / 2} ; \quad x_{\min }=\left(y^{3}-9999\right)^{1 / 2} .
$$

Thus, if $y$ is large, the possible values of $x$ in this search are severely limited.

There are two methods of finding the starting values of $x$ for a given $y$.

(1) To compute the square-root directly.

(2) To compute it by a search routine.

A routine was programmed, using the fact for $y>21, x_{\min }$ is a monotonic increasing function of $y$. This was found to be considerably quicker than (1) and was used throughout.

Results. The final output is rather large and it is intended to deposit a copy in the UMT file. A limited number of copies have been retained by the authors for distribution to interested mathematicians.

The tables contain all solutions found in ascending order of $k$ as well as a summary giving the total number of solutions for each value. 
The present paper contains in Table 1 a somewhat shortened version of that summary, and lists all values of $|k|$ for which six or more solutions were found.

We summarize some of our results as follows:

(1) For positive $k \leqq 100$, no solution could be appended to the Table in [3].

(2) For negative $k \geqq-9999$, the last solution found was

$$
(1,775,104)^{3}-(2,365,024,826)^{2}=-5412 ;
$$

whilst, for positive $k \leqq 9999$, the last solution was

$$
(939,787)^{3}-(911,054,064)^{2}=307 .
$$

(3) In addition to solutions for $|k| \leqq 9999$, we have solutions, for $y \geqq 10^{4}$ and $|k| \leqq 99999$; there are 1221 for positive $k$ and 799 for negative $k$.

The vast majority of solutions are with $y<100$ and Table 2 gives the number of solutions for various ranges of $y$.

The fact that the number of solutions is a rapidly decreasing function of $y$ suggests that for at least some $k$ the solution set may be complete.

Memorial University of Newfoundland

St. John's, Newfoundland, Canada

1. L. J. Mordell, A Chapter in the Theory of Numbers, Cambridge at the University Press and Macmillan, New York, 1947. MR 8, 502.

2. B. M. DELONE \& D. K. FADDEEv, The Theory of Irrationalities of the Third Degree, (English translation), Transl. Math. Monographs, Vol. 10, Amer. Math. Soc., Providence, R.I., 1964. MR 28 *3955.

3. O. Hemer, "Notes on the Diophantine equations $y^{2}-k=x^{3}$," Ark. Mat., v. 3, 1954, pp. 67-77. MR 15, 776.

4. W. LJungaren, "On the Diophantine equation $y^{2}-k=x^{3}$," Acta Arith., v. 8, 1963, pp. 451-463. MR 28 *2082.

\section{Experiments on Gram-Schmidt Orthogonalization}

\section{By John R. Rice*}

1. Orthogonalization Procedures. In this note we present a brief resumé of some experiments made on orthogonalization methods. We have a set $\left\{u_{i} \mid i=1,2, \cdots, n\right\}$ of $m$-vectors and wish to obtain an equivalent orthonormal set $\left\{v_{i} \mid i=1,2, \cdots, n\right\}$ of $m$-vectors. We consider the following methods:

(a) Gram-Schmidt (GS). $v_{1}=u_{1} /\left\|u_{1}\right\|$.

$$
v_{k}{ }^{\prime}=u_{k}-\sum_{j=1}^{k-1}\left(v_{j}, u_{k}\right) v_{j}, \quad v_{k}=v_{k}{ }^{\prime} /\left\|v_{k}{ }^{\prime}\right\| ; \quad k=2, \cdots, n .
$$

(b) Modified Gram-Schmidt (MGS). $v_{1}=u_{1} /\left\|u_{1}\right\|$,

$$
\left.\begin{array}{rl}
u_{j}^{(1)} & =u_{j}-\left(u_{j}, v_{1}\right) v_{1}, \quad j=2, \cdots, n . \\
v_{k} & =u_{k}{ }^{(k-1)} /\left\|u_{k}{ }^{(k-1)}\right\| \\
u_{j}{ }^{(k)} & =u_{j}^{(k-1)}-\left(u_{j}^{(k-1)}, v_{k}\right) v_{k} \quad j=k+1, \cdots, n
\end{array}\right\} k=2, \cdots, n .
$$

Received July 13, 1965. Revised August 11, 1965.

* Purdue University. 